\title{
On the Kalman-Yakubovich-Popov lemma and the multidimensional models
}

\author{
Olivier Bachelier • Wojciech Paszke • Driss Mehdi
}

Received: 10 April 2007 / Revised: 11 January 2008 / Accepted: 20 March 2008 /

Published online: 12 April 2008

(C) The Author(s) 2008

\begin{abstract}
This paper focuses on Kalman-Yakubovich-Popov lemma for multidimensional systems described by Roesser model that possibly includes both continuous and discrete dynamics. It is shown that, similarly to the standard 1-D case, this lemma can be studied through the lens of S-procedure. Furthermore, by virtue of this lemma, we will examine robust stability, bounded and positive realness of multidimensional systems.
\end{abstract}

Keywords KYP lemma $\cdot$ Hybrid $n$-D Roesser model $\cdot$ S-procedure $\cdot$ Polynomial matrix $\partial \mathcal{D}$-regularity $\cdot$ LMI

\section{Introduction}

In the past three decades, a large attention has been paid to the study of multidimensional (n-D) systems (Bose 1982, 1985; Gałkowski and Wood 2001; Kaczorek 1985). These systems are characterized by rational functions, or matrices of several independent variables which can represent different space coordinates or mixed time and space variables. This is a result of information propagation in more than one independent direction which is the essential difference from the classical, or one-dimensional (1-D) case, where information propagates only in one direction.

This work is partially supported by Programme POLONIUM.

O. Bachelier · D. Mehdi

L.A.I.I., E.S.I.P., University of Poitiers, 40 Avenue du Recteur Pineau, 86022 Poitiers Cedex, France

O. Bachelier

e-mail: Olivier.Bachelier@univ-poitiers.fr

D. Mehdi

e-mail: Driss.Mehdi@univ-poitiers.fr

W. Paszke $(\bowtie)$

Control Systems Technology Group, Eindhoven University of Technology, PO Box 513,

5600 MB Eindhoven, The Netherlands

e-mail: W.Paszke@tue.nl 
The interest in $n$-D systems has predominantly been motivated by a wide variety of applications, arising in both theory and practical applications. Particular applications include $n$-D filtering (Basu 2002; Lu and Antoniou 1992), $n$-D coding and decoding (Shi and Zhang 2002), image processing (Bracewell 1995), and multidimensional signal processing (Dudgeon and Merserau 1984). $n$-D systems theory also successfully applies for analysis and synthesis of processes with repetitive dynamics, see for example Rogers et al. (2007) and the references therein.

Two basic state-space models for $n$-D systems have been developed. The first is credited to Roesser (1975) and clearly has a first order structure. In what follows, the state vector is partitioned into sub-vectors-one for each of $n$ directions of information propagation. Another commonly used state-space model for $n$-D systems has been proposed by Fornasini and Marchesini (1978). Note, however, that these models are not fully independent and it is possible to transform one into the other.

The unquestioned popularity of the state-space methods in $n$-D system theory stems from the fact that they are well understood and efficient numerical linear algebra routines exist (required when manipulating state-space models). In what follows, the stability problem, which is a main requirement for $n$-D systems, can be solved within Lyapunov's framework (Gałkowski et al. 2003b; Hinamoto 1993; Kaczorek 1985; Lu 1994), which is naturally performed in the state space. The most important fact associated with such an approach is that an $n$-D system stability condition can be recast into a linear matrix inequality (LMI) feasibility problem (Boyd etal. 1994) i.e. finite dimensional convex optimization problem involving LMI constraints. It has to be mentioned that most of the proposed LMI stability conditions, when tractable, are only sufficient. Recently, some preliminary results on formulating necessary and sufficient stability condition have been proposed in Ebihara et al. (2006), Fu et al. (2006). However, these conditions are only formulated for 2-D discrete system case and cannot be easily extended to controller design case.

However, the most known conditions relevant to the stability of $n$-D systems are expressed in terms of characteristic polynomial root-clustering (Jury 1978). In this framework, it becomes of interest to derive theoretical results on polynomial matrices (Šebek 1988). Once again, convex optimization techniques over LMI constraints have turned to be a good tool to tackle some problems induced by polynomial matrices, especially $\mathcal{D}$-stability analysis (Henrion et al. 2001). This paper lies in this framework.

The connection between the polynomial, or, in a more restrictive way, frequency approach, and the matrix inequalities is quite well established in the context of 1-D systems. The strongest result is the celebrated Kalman-Yakubovich-Popov (KYP) lemma (Rantzer 1996; Iwasaki and Hara 2005) which gives equivalences between crucial frequency domain inequalities and LMIs.

To date, no work has been reported on a solution to this problem in terms of $n$-D systems and therefore it is a natural question to ask if it is possible to provide a version of the KYP lemma which can be exploited in the realm of $n$-D systems. Recently, much effort has been dedicated to establishing KYP lemma for $n$-D systems. Although few special instances of the KYP lemma have been implicitly addressed for 2-D models (Xu et al. 2003, 2005), no real general result has been provided yet. This paper aims at filling this gap.

The paper is organized as follows. After this introduction, Sect. 2 provides the preliminary background. It recalls the Roesser model and shortly highlights its various extensions. These models are very popular to describe the behaviour of multidimensional systems. Besides, it exploits some descriptions (encountered in the literature) of regions of the complex plane as well as the notion of $\partial \mathcal{D}$-regularity for multivariate matrix functions. Section 3 states the main result which is some extension of the generalized KYP lemma. In Sect.4, this lemma 
is applied to analyze the behaviour of $n$-D systems described with Roesser model introduced in Sect. 2. Some numerical illustration is provided in Sect. 5 to highlight the relevance of the approach. Finally, results are summarized and conclusions are stated in Sect. 6, where a particular emphasis is put on possible further investigations.

The following notation will be used throughout the paper. $M^{\prime}$ denotes the transpose conjugate of a matrix $M$. Hence, $\lambda^{\prime}$ is the conjugate of complex number $\lambda$. $\|M\|_{2}$ is the matrix 2 -norm (the maximum singular value) induced by the Euclidean vector norm. $I_{n}$ is the identity matrix of dimension $n$ and $I$ (resp. 0 ) is the identity (resp. a null) matrix of appropriate dimensions. Matrix inequalities are considered in the sense of Löwner, i.e. $>0$ (resp. $<0$ ) means positive (resp. negative) definite and $\geq 0$ (resp. $\leq 0)$ means positive (resp. negative) semi-definite. The notation $\mathcal{H}_{n}$ stands for the set of Hermitian matrices of dimension $n$. $\mathcal{H}_{n}^{+} \subset \mathcal{H}_{n}$ is the subset of positive definite matrices and $\mathcal{H}_{n}^{-} \subset \mathcal{H}_{n}$ that of negative definite matrices. Also let the following notations be defined:

$$
\bigoplus_{i=1}^{k} M_{i}=\underset{i=1, \ldots k}{\operatorname{diag}}\left\{M_{i}\right\}
$$

For a given subset $\mathcal{S}$ of the set $\mathcal{X}$, the set $\mathcal{S}^{C}$ is the complementary set of $\mathcal{S}$ such that $\mathcal{S} \cup \mathcal{S}^{C}=\mathcal{X} \& \mathcal{S} \cap \mathcal{S}^{C}=\emptyset$. At last, the sets of indices are denoted as follows:

$$
\mathbb{I}(q):=\{1, \ldots, q\}, \quad q \in \mathbb{N} .
$$

\section{Preliminaries}

This section introduces some preliminaries, some of them being borrowed from the literature. The first part is dedicated to the presentation of the Roesser models and to some associated stability conditions. The second provides the description of a class of regions of the "multidimensional" complex plane which will be used in the next section. The third part briefly introduces the notion of $\partial \mathcal{D}$-regularity of a multivariate matrix function, inspired from the concept of matrix $\partial \mathcal{D}$-regularity.

\subsection{Hybrid Roesser model}

One of the most commonly used model for $n$-D discrete systems is the Roesser model (RM) that has been originally introduced in Roesser (1975). One of the key features of this model is that the state vector is partitioned into horizontal and vertical components.

Furthermore, it is also possible to define the continuous or hybrid versions of Roesser model. The general version of the hybrid Roesser model for $n$-D systems takes the following form (Bochniak 2005)

$$
\begin{gathered}
{\left[\begin{array}{c}
\frac{\partial}{\partial t_{1}} x^{1}\left(t_{1}, \ldots, t_{r}, j_{r+1}, \ldots, j_{k}\right) \\
\vdots \\
\frac{\frac{\partial}{\partial t_{r}} x^{r}\left(t_{1}, \ldots, t_{r}, j_{r+1}, \ldots, j_{k}\right)}{x^{r+1}\left(t_{1}, \ldots, t_{r}, j_{r+1}+1, \ldots, j_{k}\right)} \\
\vdots \\
x^{k}\left(t_{1}, \ldots, t_{r}, j_{r+1}, \ldots, j_{k}+1\right)
\end{array}\right]=} \\
+\left[\begin{array}{c|c}
A_{11} & A_{12} \\
\hline A_{21} \mid A_{22}
\end{array}\right]\left[\begin{array}{c}
x^{1}\left(t_{1}, \ldots, t_{r}, j_{r+1}, \ldots, j_{k}\right) \\
\vdots \\
\frac{x^{r}\left(t_{1}, \ldots, t_{r}, j_{r+1}, \ldots, j_{k}\right)}{x^{r+1}\left(t_{1}, \ldots, t_{r}, j_{r+1}, \ldots, j_{k}\right)} \\
\vdots \\
x^{k}\left(t_{1}, \ldots, t_{r}, j_{r+1}, \ldots, j_{k}\right)
\end{array}\right] \\
+\left[\frac{B_{1}}{B_{2}}\right] u\left(t_{1}, \ldots, t_{r}, j_{r+1}, \ldots, j_{k}\right)
\end{gathered}
$$




$$
y\left(t_{1}, \ldots, t_{r}, j_{r+1}, \ldots, j_{k}\right)=\left[C_{1} \mid C_{2}\right]\left[\begin{array}{c}
x^{1}\left(t_{1}, \ldots, t_{r}, j_{r+1}, \ldots, j_{k}\right) \\
\vdots \\
\frac{x^{r}\left(t_{1}, \ldots, t_{r}, j_{r+1}, \ldots, j_{k}\right)}{x^{r+1}\left(t_{1}, \ldots, t_{r}, j_{r+1}, \ldots, j_{k}\right)} \\
\vdots \\
x^{k}\left(t_{1}, \ldots, t_{r}, j_{r+1}, \ldots, j_{k}\right)
\end{array}\right]
$$

where $\sum_{i=1}^{k} n_{i}=n$.

The vectors $x^{i}\left(t_{1}, \ldots, t_{r}, j_{r+1}, \ldots, j_{k}\right) \in \mathbb{R}^{n_{i}}, i=1, . ., k, u\left(t_{1}, \ldots, t_{r}, j_{r+1}, \ldots, j_{k}\right) \in$ $\mathbb{R}^{m}$ and $y\left(t_{1}, \ldots, t_{r}, j_{r+1}, \ldots, j_{k}\right) \in \mathbb{R}^{p}$ are the local state subvectors, the input vector and the output vector respectively. The matrices

$$
\begin{aligned}
& A=\left[\begin{array}{l|l}
A_{11} & A_{12} \\
\hline A_{21} & A_{22}
\end{array}\right] \in \mathbb{R}^{n \times n}, \quad B=\left[\frac{B_{1}}{B_{2}}\right] \in \mathbb{R}^{n \times m}, \\
& C=\left[C_{1} \mid C_{2}\right] \in \mathbb{R}^{p \times n}, \quad D \in \mathbb{R}^{p \times m}
\end{aligned}
$$

are the state, control, observation and direct transfer matrices respectively. Obviously, the systems represented by the above model have continuous dynamics along $r$ dimensions and discrete dynamics along $(k-r)$ dimensions. If $r=0$, then it reduces to the classical Roesser model (1975) whereas if $r=k$ then one gets its continuous counterpart. At last, if $r=1$ and $k=2$, then the obtained 2-D model is particularly suitable to describe the differential repetitive processes (Gałkowski et al. 2003c).

Note that relationships between polynomial matrix theory and state-space description are very strong in the $n$-D linear case. By applying the Laplace transform and the $\mathcal{Z}$-transform, the following frequency characterization of the system (1) is obtained

$$
Y(\lambda)=G(\lambda) U(\lambda),
$$

where

$$
G(\lambda)=C(H(\lambda)-A)^{-1} B+D, \quad H(\lambda):=\bigoplus_{i=1}^{k} \lambda_{i} I_{n_{i}}
$$

Now let the function $c(\lambda, A)$ be defined by

$$
c(\lambda, A):=\operatorname{det}(H(\lambda)-A) .
$$

We begin with presenting the stability condition for $n$-D systems, which is just a reformulation of a proposition in Bochniak (2005).

Lemma 1 Consider a multidimensional system represented by (1). Then, such a system is asymptotically stable if and only if

$$
c(\lambda, A) \neq 0 \quad \forall \lambda \in \mathcal{S}^{C},
$$

where $c(.,$.$) is defined by (3) and where \mathcal{S}^{C}$, the complementary set of the "asymptotic stability region", is defined by

$$
\mathcal{S}^{C}=\left\{\lambda=\left[\begin{array}{c}
\lambda_{1} \\
\vdots \\
\lambda_{k}
\end{array}\right] \in \mathbb{C}^{k}: \operatorname{Re}\left(\lambda_{i}\right) \geq 0, i=1, \ldots, r \&\left|\lambda_{i}\right| \geq 1, i=r+1, \ldots, k\right\} .
$$


It can be seen that the above lemma covers the existing results for both discrete and continuous $n$-D systems. Obviously, if $r=0$, then the condition for the stability of a discrete Roesser model proposed in Agathoklis (1988), Jury (1978) is recovered. Furthermore, by taking $r=k$ the result of Piekarski (1977) is obtained.

In Lemma 1, the stability condition is expressed in terms of polynomial root-clustering which generally does not lead to computationally feasible conditions. One of the ways to obtain stability conditions that result in a significant reduction in computational complexity is to formulate them as convex optimization problems involving LMIs. Therefore, the next part provides some preliminaries on point-clustering in order to prepare the derivation of some LMI-based conditions in the next section.

\subsection{Point-clustering}

Unlike the 1-D system case, zeros of $n$-D system characteristic polynomial (i.e. system poles) are not isolated and generally they cannot be a finite set. Furthermore, it is accompanied by difficulties in applying the pole placement technique for $n$-D systems, since there is no link between pole location and the dynamic response of $n$-D system. Therefore, it becomes clear that, as clustering regions, only the imaginary axis and the unit circle, which are the boundaries of $\mathcal{S}^{C}$ defined in (5), can be considered.

To proceed, consider the following matrices

$$
\left[\begin{array}{ll}
R_{i_{11}} & R_{i_{10}}^{\prime} \\
R_{i_{10}} & R_{i_{00}}
\end{array}\right] \in \mathbb{C}^{2 \times 2} .
$$

Let the sets $\partial \mathcal{D}_{i}$ be described by

$$
\partial \mathcal{D}_{i}:=\left\{s \in \mathbb{C}: \mathcal{F}_{R_{i}}(s)=0, \quad \forall i \in \mathbb{I}(k)\right\}
$$

where the functions $\mathcal{F}_{R_{i}}(s)$ are defined by

$$
\mathcal{F}_{R_{i}}(s):=\left[\begin{array}{c}
s I \\
I
\end{array}\right]^{\prime} R_{i}\left[\begin{array}{c}
s I \\
I
\end{array}\right] \quad \forall i \in \mathbb{I}(k) .
$$

Due to the fact that the only meaningful sets are lines or circles we limit our consideration to sets described by the equalities $\mathcal{F}_{R_{i}}(s)=0$.

Now define the " $k$-region" $\partial \mathcal{D}$ as

$$
\partial \mathcal{D}:=\partial \mathcal{D}_{1} \times \partial \mathcal{D}_{2} \times \cdots \times \partial \mathcal{D}_{k} .
$$

In the remaining part of the paper, we shall restrict the study to matrices that comply with

$$
R_{i}=\left[\begin{array}{ll}
0 & 1 \\
1 & 0
\end{array}\right] \quad \forall i \in \mathbb{I}(r) \text { and }\left[\begin{array}{rr}
1 & 0 \\
0 & -1
\end{array}\right] \quad \forall i \in\{r+1, \ldots, k\},
$$

meaning that, respectively, only the imaginary axis and the unit circle are considered as instances of $\partial \mathcal{D}_{i}$. Indeed, as it has been mentioned, they are the ones of practical interest for the study of $n \mathrm{D}$ models. More precisely, with such a choice, $\partial \mathcal{D}$ is the boundary of $\mathcal{S}^{C}$ defined in (5). But note that another work considering a much larger class of regions has been completed in a technical report which is available upon request to the authors.

Let $\lambda$ be a complex vector that can be written

$$
\lambda:=\left[\begin{array}{c}
\lambda_{1} \\
\vdots \\
\lambda_{k}
\end{array}\right] \in \mathbb{C}^{k} .
$$


In the sequel, define the following matrices

$$
\begin{aligned}
& \Lambda:=\bigoplus_{i=1}^{k} \lambda_{i} ;
\end{aligned}
$$

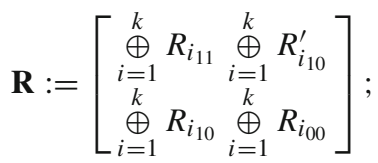

to obtain

$$
\lambda \in \partial \mathcal{D} \Leftrightarrow\left[\begin{array}{c}
\Lambda \\
I_{k}
\end{array}\right]^{\prime} \mathbf{R}\left[\begin{array}{c}
\Lambda \\
I_{k}
\end{array}\right]=0 .
$$

Before proceeding further, we give the following lemma which will be useful in the sequel.

Lemma 2 Let a $k$-region $\partial \mathcal{D}$ be defined as in (9) with (6)-(8), (10) and $\lambda \in \boldsymbol{C}^{k}$ comply with (11). The two following statements are equivalent.

(i)

$$
\lambda \in \partial \mathcal{D}
$$

(ii)

$$
\left[\begin{array}{c}
H(\lambda) \\
I
\end{array}\right]^{\prime} \overline{\mathbf{R}}(\mathcal{P})\left[\begin{array}{c}
H(\lambda) \\
I
\end{array}\right]=0 \quad \forall \mathcal{P} \in \mathbb{H}
$$

where $\mathbb{H}=\left\{\mathcal{H}_{n_{1}} \times \cdots \times \mathcal{H}_{n_{k}}\right\}, H(\lambda)$ is defined in (2) and $\overline{\mathbf{R}}(\mathcal{P})$ stands for

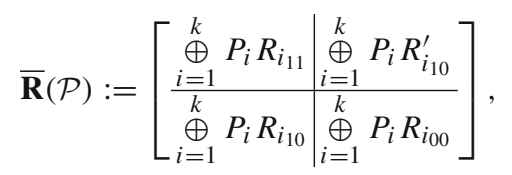

and with $P_{n_{i}} \in \mathcal{H}_{n_{i}}, \quad \forall i \in \mathbb{I}(k)$.

Proof (i) $\Rightarrow$ (ii): if $\lambda \in \partial \mathcal{D}$ then the equality in (12) holds or equivalently

$$
\bigoplus_{i=1}^{k} \mathcal{F}_{R_{i}}\left(\lambda_{i}\right)=0
$$

leading to

$$
\bigoplus_{i=1}^{k} \mathcal{F}_{R_{i}}\left(\lambda_{i}\right) P_{i}=0
$$

with considering any set $\mathcal{P}$ of $k$ matrices $P_{i} \in \mathcal{H}_{n_{i}}, i \in \mathbb{I}(k)$, one gets 


$$
\left[\begin{array}{c}
H(\lambda) \\
I
\end{array}\right]^{\prime} \overline{\mathbf{R}}(\mathcal{P})\left[\begin{array}{c}
H(\lambda) \\
I
\end{array}\right]=0 .
$$

(ii) $\Rightarrow$ (i): Assume that (14) holds. Let a vector $z$ comply with

$$
z=\left[\begin{array}{c}
z_{1} \\
\vdots \\
z_{k}
\end{array}\right] \quad \text { with } \quad z_{i} \in \mathbb{C}^{n_{i}}, \quad \forall i \in \mathbb{I}(k) .
$$

Left and right multiplying (14) by $z^{\prime}$ and $z$ respectively yields

$$
\sum_{i=1}^{k}\left(\left(z_{i}^{\prime} P_{i} z_{i}\right) \mathcal{F}_{R_{i}}\left(\lambda_{i}\right)\right)=0 .
$$

The above inequality holds for any set $\mathcal{P}$ meaning that $z_{i}^{\prime} P_{i} z_{i}$ can take any value. Thus, it is clear that $\mathcal{F}_{R_{i}}\left(\lambda_{i}\right)=0 \quad \forall i \in \mathbb{I}(k)$.

\subsection{Multivariate matrix $\partial \mathcal{D}$-regularity}

To conclude this section, the definition of the $\partial \mathcal{D}$-regularity of a multivariate matrix function is proposed, accompanied by a short discussion.

Definition 1 Let $\partial \mathcal{D}$ be a subset of $\mathbb{C}^{k}$ and $\lambda \in \mathbb{C}^{k}$ comply with (11). Also consider a $k$-variate matrix function $\mathbf{A}(\lambda)$. Then, $\mathbf{A}(\lambda)$ is said to be

- $\partial \mathcal{D}$-regular if $\left\{\lambda \in \mathbb{C}^{k}: \operatorname{det}(\mathbf{A}(\lambda))=0\right\} \cap \partial \mathcal{D}=\emptyset$;

- $\partial \mathcal{D}$-singular if $\left\{\lambda \in \mathbb{C}^{k}: \operatorname{det}(\mathbf{A}(\lambda))=0\right\} \cap \partial \mathcal{D} \neq \emptyset$.

In Sect. 4, a particular attention will be paid to the $k$-D polynomial matrix of the form

$$
\mathbf{A}(\lambda):=E H(\lambda)-A .
$$

In this case, and if $E=I$, one gets $\operatorname{det}(\mathbf{A}(\lambda))=c(\lambda, A)$ which corresponds to the characteristic polynomial of Roesser model (1). Thus, from Lemma 1, it can be seen that the asymptotic stability of (1) is a special instance of the $\partial \mathcal{D}$-regularity of $\mathbf{A}(\lambda)$ as defined in (20). More precisely, for (1) to be asymptotically stable, it is necessary that $\mathbf{A}(\lambda)$ be $\partial \mathcal{D}$-regular with (6)-(10).

In such a case, the difference between $\partial \mathcal{D}$-regularity and asymptotic stability lies in the distribution of the roots of $c(\lambda, A)$ with respect to $\partial \mathcal{D}$. More generally, $\mathcal{D}$-stability requires $\partial \mathcal{D}$-regularity when $\partial \mathcal{D}$ is the boundary of the region $\mathcal{D}$.

For the classical 1-D case $(r=k=1$ or $k=k-r=1)$, then $\mathbf{A}(\lambda)$ can be written $\mathbf{A}(\lambda)=(\lambda E-A)$ and, if $E=I$, Definition 1 becomes equivalent to the definition of the $\partial \mathcal{D}$-regularity of a matrix $A$ proposed in Bachelier et al. (2004). If $E \neq I$, then the definition in Bachelier et al. (2004) can be directly extended to the notion of $\partial \mathcal{D}$-regularity of the pencil $(E, A)$.

\section{A version of the KYP lemma}

This section is devoted to the derivation of the main result which is some sort of $k$-D version of the celebrated KYP-lemma. This version will only provide a sufficient condition but the 
necessity will be briefly discussed with the help of the so-called generalized S-procedure. A large reference is made to the seminal paper (Iwasaki and Hara 2005) where generalized KYP lemma for 1-D linear models and the generalized S-procedure are presented in a very elegant way.

Before stating the result, let us introduce the following notation useful for a further analysis (note that various notations are borrowed from Iwasaki and Hara 2005).

Consider some matrix $F \in \mathbb{C}^{(n+m) \times(n+m)}$ and let another matrix $\Theta$ belong to $\mathcal{H}_{n+m}$. Next, let the set $\mathcal{P}$ be made up by $k$ matrices $P_{i} \in \mathcal{H}_{n_{i}}, \quad \forall i \in \mathbb{I}(k)$. With each set $\mathcal{P}$ and with a set $\partial \mathcal{D}$ given by (9) with (6)-(8) and (10), one can associate a matrix $\overline{\mathbf{R}}(\mathcal{P})$, as in Lemma 2, defined by (15). The set $\mathbf{M}$ is the set of all complex matrices $M$ associated with $F$ and $\overline{\mathbf{R}}(\mathcal{P})$ in the following way:

$$
\mathbf{M}:=\left\{M \in \mathcal{H}_{n+m}: M=F^{\prime} \overline{\mathbf{R}}(\mathcal{P}) F\right\} .
$$

From the above formulation, the subset $\tilde{\mathbf{M}}$ is defined as

$$
\tilde{\mathbf{M}}:=\left\{M \in \mathbf{M}:(M+\Theta) \in \mathcal{H}_{n+m}^{-}\right\} .
$$

At last, for a complex vector $\lambda$ defined as in (11), the matrices $\Gamma(\lambda)$ and $N(\lambda)$ are defined by

$$
\Gamma(\lambda):=[I-H(\lambda)] \text { and } \operatorname{Span}(N(\lambda)):=\operatorname{Ker}(\Gamma(\lambda) F),
$$

respectively.

Now, the main result, which can be considered as some extension of the KYP lemma in its "strict inequality" version (Rantzer 1996) is stated.

Theorem 1 With the notations and assumptions detailed above, consider the two following statements:

(i)

$$
N^{\prime}(\lambda) \Theta N(\lambda) \in \mathcal{H}_{m}^{-} \quad \forall \lambda \in \partial \mathcal{D}
$$

(ii)

$$
\tilde{\mathbf{M}} \neq \emptyset .
$$

Then (ii) is sufficient for (i).

Before giving the proof to the above theorem, some comments on the two conditions are provided. The first statement corresponds to some property to be checked such as robust stability, $\mathcal{H}_{\infty}$ performance level, and others (see subsection 4.1.1 to understand how a suitable choice of $\Theta$ can link $N(\lambda)$ and $\Theta$ to a property to be satisfied by the system transfer matrix). The second statement corresponds to some numerically tractable condition which simply means that there exists some set $\mathcal{P}$ (which is usually seen as a set of Lyapunov matrices), such that

$$
M+\Theta<0 .
$$

The idea is then to handle inequality (25) rather than directly tackle the original property (i) which cannot be easily exploited from a numerical point of view.

Proof Firstly, observe that (24), as mentioned above, means that there exists a set $\mathcal{P}$ such that (25) holds. Further, based on the definition of $N(\lambda)$, it can be seen that

$$
\Gamma(\lambda) F N(\lambda)=0, \quad \forall \lambda \in \partial \mathcal{D} .
$$


Next, inequality (25) implies that

$$
N^{\prime}(\lambda)(M+\Theta) N(\lambda)<0, \quad \forall \lambda \in \partial \mathcal{D}
$$

and hence

$$
N^{\prime}(\lambda) F^{\prime} \overline{\mathbf{R}}(\mathcal{P}) F N(\lambda)+N^{\prime}(\lambda) \Theta N(\lambda)<0, \quad \forall \lambda \in \partial \mathcal{D} .
$$

Moreover, we can see from (26) that $\operatorname{Span}(F N(\lambda))$ is completely characterized by the relation

$$
\operatorname{Span}(F N(\lambda))=\operatorname{Span}\left(\left[\begin{array}{c}
H(\lambda) \\
I
\end{array}\right] X\right),
$$

where $X$ is any full rank matrix. Thus, inequality (28) can be equivalently written as

$$
X^{\prime}\left[\begin{array}{c}
H(\Lambda) \\
I
\end{array}\right]^{\prime} \overline{\mathbf{R}}(\mathcal{P})\left[\begin{array}{c}
H(\Lambda) \\
I
\end{array}\right] X+X^{\prime} N^{\prime}(\lambda) \Theta N(\lambda) X<0, \quad \forall \lambda \in \partial \mathcal{D} .
$$

Finally, taking the result of Lemma 2 into account, it is clear that the first term of the left handside member of inequality (30) is zero when $\lambda \in \partial \mathcal{D}$. Hence, the second term is negative definite which is equivalent to (i). This completes the proof.

The important point to note is that the 1-D version of KYP lemma is known to provide a necessary and sufficient condition whereas Theorem 1 only proposes a sufficient condition (ii) for property (i) to hold. More than proving Theorem 1, it is important to emphasize why condition (ii) might not be necessary. The 1-D version of KYP lemma (Rantzer 1996) can be proven through the so-called S-procedure (Yakubovich 1971) in its generalized form (see Iwasaki and Hara (2005) and the references therein). In the remaining part of the section, Theorem 1 is studied through the lens of S-procedure, which is now recalled in its generalized strict inequality version.

Lemma 3 (S-procedure, from Iwasaki and Hara 2005) Let $\boldsymbol{\Theta}$ be an Hermitian matrix and $\mathbf{M}$ be an arbitrary subset of $\mathcal{H}_{q}$. Moreover let $\tilde{\mathbf{M}} \subset \mathbf{M}$ be defined by

$$
\tilde{\mathbf{M}}:=\left\{M \in \mathbf{M}:(M+\Theta) \in \mathcal{H}_{q}^{-}\right\}
$$

and the set $\mathbf{S}$ be defined by

$$
\mathbf{S}:=\left\{S \in \mathcal{H}_{q}: S \neq 0, \operatorname{rank}(S)=1, S \geq 0, \operatorname{tr}(M S) \geq 0 \quad \forall M \in \mathbf{M}\right\} .
$$

Consider the two following statements:

(a)

$$
\operatorname{tr}(\boldsymbol{\Theta} S)<0, \quad \forall S \in \mathbf{S}
$$

(b)

$$
\tilde{\mathbf{M}} \neq \varnothing .
$$

Then (b) is sufficient for (a) and if the set $\mathbf{M}$ is rank-one separable (see the definition in Iwasaki and Hara 2005) then (b) is also necessary and the S-procedure is said lossless.

A possible approach to understand why Theorem 1 only provides a sufficient condition unlike the classical KYP lemma is to try to see why S-procedure is either useless or is simply not lossless when applied to the assumptions of Theorem 1. According to the used notations, it is clear that the idea is to compare the sets $\mathbf{M}$ and $\tilde{\mathbf{M}}$ defined, on one hand, in Theorem 1 and, on the other hand, in Lemma 3 with $q=n+m$, and then to address two issues. 
1. Is it possible to exhibit a set $\mathbf{S}=\mathbf{S}_{a}$ from the assumptions of Theorem 1 that could match the set $\mathbf{S}=\mathbf{S}_{b}$ in Lemma 3 (this would enable ones to apply the S-procedure, at least in the sense $\mathbf{b}) \Rightarrow \mathbf{a}))$ ?

2. If so, is the S-procedure lossless (this would enable ones to apply the S-procedure in the sense $\mathbf{a}) \Rightarrow \mathbf{b}))$ ?

Firstly, it can be seen that the statement (i) in Theorem 1 is equivalent to

$$
y^{\prime} N^{\prime}(\lambda) \Theta N(\lambda) y<0, \quad \forall y \neq 0, \quad \forall \lambda \in \partial \mathcal{D} .
$$

Next, substitute $\xi=N(\lambda) y$ into (35) to obtain

$$
\operatorname{tr}(\Theta S)<0, \quad \forall S \in \mathbf{S}_{a}:=\left\{S=\xi \xi^{\prime}: \xi \neq 0, \Gamma(\lambda) F \xi=0, \lambda \in \partial \mathcal{D}\right\} .
$$

With the change $\eta=F \xi$, it is easy to see that $\eta$ complies with

$$
\eta=\left[\begin{array}{c}
H(\lambda) \\
I
\end{array}\right] z, z \neq 0,
$$

and hence the set $\mathbf{S}_{a}$ can be written

$$
\mathbf{S}_{a}=\left\{\xi \xi^{\prime}: \xi \neq 0, \eta=F \xi=\left[\begin{array}{c}
H(\lambda) \\
I
\end{array}\right] z, \lambda \in \partial \mathcal{D}\right\} .
$$

In the following, based on Lemma 2, it can be deduced that

$$
\begin{aligned}
\mathbf{S}_{a}= & \left\{\xi \xi^{\prime}: \xi \neq 0, \eta=F \xi=\left[\begin{array}{c}
H(\lambda) \\
I
\end{array}\right] z,\right. \\
& {\left.\left[\begin{array}{c}
H(\lambda) \\
I
\end{array}\right]^{\prime} \overline{\mathbf{R}}(\mathcal{P})\left[\begin{array}{c}
H(\lambda) \\
I
\end{array}\right]=0 \quad \forall \mathcal{P} \in \mathbb{H}\right\} . } \\
\Leftrightarrow \mathbf{S}_{a}= & \left\{\xi \xi^{\prime}: \xi \neq 0, \eta=F \xi=\left[\begin{array}{c}
H(\lambda) \\
I
\end{array}\right] z,\right. \\
& \left.q^{\prime}\left[\begin{array}{c}
H(\lambda) \\
I
\end{array}\right]^{\prime} \overline{\mathbf{R}}(\mathcal{P})\left[\begin{array}{c}
H(\lambda) \\
I
\end{array}\right] q=0 \quad \forall\{\mathcal{P} ; q\} \in \mathbb{H} \times \mathbb{C}^{n}\right\} .
\end{aligned}
$$

so, it is possible to express (i) as (a) with $\Theta=\Theta$ and with $\mathbf{S}$ matching $\mathbf{S}_{a}$ as described above.

Secondly, the condition (ii) in Theorem 1 can be expressed as (b) in Lemma 3 with $\Theta=\Theta$ and with the set $\mathbf{M}$ as defined in (21). Next, application of Lemma 3 yields

$$
\operatorname{tr}(\Theta S)<0, \quad \forall S \in \mathbf{S}_{b}:=\left\{S=\xi \xi^{\prime}: \xi \neq 0, \operatorname{tr}\left(M \xi \xi^{\prime}\right) \geq 0 \quad \forall M \in \mathbf{M}\right\},
$$

where $\mathbf{S}_{b}$ can also be written as

$$
\mathbf{S}_{b}=\left\{S=\xi \xi^{\prime}: \xi \neq 0, \xi^{\prime} F^{\prime} \overline{\mathbf{R}}(\mathcal{P}) F \xi \geq 0 \quad \forall \mathcal{P} \in \mathbb{H}\right\}
$$

Now, since $\mathcal{P}$ is any set in $\mathbb{H}$ then the inequality involved in the above description of $\mathbf{S}_{b}$ holds for any choice $\mathcal{P}_{+}=\left\{P_{i}\right\} \in \mathbb{H}$ as well as for any choice $\mathcal{P}_{-}=\left\{-P_{i}\right\} \in \mathbb{H}$. In the following, observe that $\overline{\mathbf{R}}(\mathcal{P})$ is linear with respect to various matrices $P_{i}$ and therefore the description of $\mathbf{S}_{b}$ can be modified as follows

$$
\mathbf{S}_{b}=\left\{S=\xi \xi^{\prime}: \xi \neq 0, \xi^{\prime} F^{\prime} \overline{\mathbf{R}}(\mathcal{P}) F \xi=0 \quad \forall \mathcal{P} \in \mathbb{H}\right\}
$$


Now, the issue 1 mentioned above is to compare $\mathbf{S}_{a}$ in (40) and $\mathbf{S}_{b}$ in (42). $\mathbf{S}_{a}$ can be written

$$
\begin{aligned}
\Leftrightarrow \mathbf{S}_{a}= & \left\{\xi \xi^{\prime}: \xi \neq 0: \eta=F \xi=\left[\begin{array}{c}
H(\lambda) \\
I
\end{array}\right] z,\right. \\
& \left.q^{\prime}\left[\begin{array}{c}
H(\lambda) \\
I
\end{array}\right]^{\prime} \overline{\mathbf{R}}(\mathcal{P})\left[\begin{array}{c}
H(\lambda) \\
I
\end{array}\right] q=0 \quad \forall\{\mathcal{P} ; q\} \in \mathbb{H} \times \mathbb{C}^{n}\right\} .
\end{aligned}
$$

which, with $q=X z$, also writes ( $X$ being any full rank matrix)

$$
\begin{aligned}
\Leftrightarrow \mathbf{S}_{a}= & \left\{\xi \xi^{\prime}: \xi \neq 0: \eta=F \xi=\left[\begin{array}{c}
H(\lambda) \\
I
\end{array}\right] z,\right. \\
& \left.z^{\prime} X^{\prime}\left[\begin{array}{c}
H(\lambda) \\
I
\end{array}\right]^{\prime} \overline{\mathbf{R}}(\mathcal{P})\left[\begin{array}{c}
H(\lambda) \\
I
\end{array}\right] X z=0 \quad \forall\{\mathcal{P} ; X: \operatorname{det}(X) \neq 0\} \in \mathbb{H} \times \mathcal{H}_{n}\right\} .
\end{aligned}
$$

Now, assume that $k=1$ which corresponds to the 1-D system case, then, with no loss of generality, the matrix $P_{1}$ can be substituted with $X^{-1} P_{1} X^{-1}$ and $\mathbf{S}_{a}$ can then be described by

$$
\begin{aligned}
\Leftrightarrow \mathbf{S}_{a}= & \left\{\xi \xi^{\prime}: \xi \neq 0: \eta=F \xi=\left[\begin{array}{c}
H(\lambda) \\
I
\end{array}\right] z,\right. \\
& \left.z^{\prime}\left[\begin{array}{c}
H(\lambda) \\
I
\end{array}\right]^{\prime} \overline{\mathbf{R}}(\mathcal{P})\left[\begin{array}{c}
H(\lambda) \\
I
\end{array}\right] z=0 \quad \forall \mathcal{P} \in \mathbb{H}\right\},
\end{aligned}
$$

which is simplified in

$$
\Leftrightarrow \mathbf{S}_{a}=\left\{\xi \xi^{\prime}: \xi \neq 0: \eta=F \xi=\left[\begin{array}{c}
H(\lambda) \\
I
\end{array}\right] z, \eta^{\prime} \overline{\mathbf{R}}(\mathcal{P}) \eta=0 \quad \forall \mathcal{P} \in \mathbb{H}\right\} .
$$

or equivalently in $\mathbf{S}_{b}$. But this equality $\mathbf{S}_{a}=\mathbf{S}_{b}$ holds when $k=1$. It means that the S-procedure can be directly applied from (ii) to (i) to prove Theorem 1 only when one comes back to the 1-D case. In this case only, shall one ask the question of necessity i.e. of the losslessness of the S-procedure. So it is lossless if the set $\mathbf{M}$ defined in (21) is rank-one separable. Actually this set is the one studied in Iwasaki and Hara (2005) so the answer is yes, it is rank-one separable.

As a summary of the above discussion, here are three points:

- Theorem 1 provides a sufficient condition that is generally not necessary.

- $\mathbf{S}_{a} \subset \mathbf{S}_{b}$, so (b) is clearly more constraining than (a); Thus, (b) $\Rightarrow$ (a). But it is not a direct application of the S-procedure. However (ii) $\Leftrightarrow$ (b) with $\mathbf{S}=\mathbf{S}_{b} \Rightarrow$ (b) with $\mathbf{S}=\mathbf{S}_{a} \stackrel{\text { S-procedure }}{\Rightarrow}$ (a) with $\mathbf{S}=\mathbf{S}_{a} \Leftrightarrow$ (i). Hence, (ii) $\Rightarrow$ (i).

- At last, when $k=1$ (1-D case), then $\mathbf{S}=\mathbf{S}_{a}=\mathbf{S}_{b}$ which enables ones to directly apply the S-procedure. Moreover, it has been proven that it is lossless in this case so (ii) $\Leftrightarrow$ (i). One recovers the classical KYP lemma (Rantzer 1996).

\section{Application to the analysis of Roesser models}

In this section, the relevance of Theorem 1 for the study of multidimensional hybrid statespace Roesser model (1) is emphasized. The idea is simply to make a suitable choice of matrices $F$ and $\theta$. Matrix $F$ is chosen as follows:

$$
F:=\left[\begin{array}{cc}
A & B \\
E & 0
\end{array}\right] \in \mathbb{R}^{n \times n},
$$


where the matrices $A, B$ are those involved in (1). The only restriction in this section is that $E$ is blockdiagonal, i.e. $E=\bigoplus_{i=1}^{k} E_{i}$ with $E_{i} \in \mathbb{R}^{n_{i} \times n_{i}}$. It can be noticed that matrix $F$ is here real which also corresponds to a special case of Theorem 1. Condition (i) in Theorem 1 must be considered as a performance level or a property of (1) to be tested. In the sequel, three properties are considered, depending on the choice of $\Theta$.

\subsection{Robust $\partial \mathcal{D}$-regularity against an LFT-based uncertainty}

This part is devoted to the analysis of the $\partial \mathcal{D}$-regularity of the uncertain $k$-variate polynomial matrix described by

$$
\mathbb{A}(\lambda)=\mathbf{A}(\lambda)-\bar{A}=E H(\lambda)-A_{c},
$$

where $\mathbf{A}(\lambda)$ is given by (20), where $A, B, C$, and $E$ have the same dimensions as those involved in (1), where $A_{c}=A+\bar{A}$ and where $\bar{A}$, given by

$$
\bar{A}=B \bar{\Delta} C,
$$

is an uncertain matrix complying with the so-called Linear Fractional Transform (LFT)-based structure:

$$
\bar{\Delta}=\Delta\left(I_{p}-D \Delta\right)^{-1}, \quad \operatorname{det}\left(I_{p}-D \Delta\right) \neq 0 .
$$

The above full rank assumption is referred to as the well posedness of the uncertainty. Matrix $\Delta$ is the actual uncertainty matrix which belongs to $\mathcal{B}(\rho)$, the ball of complex matrices $\Delta$ verifying $\|\Delta\|_{2} \leq \rho$.

\subsubsection{Complex $\partial \mathcal{D}$-regularity radius}

Nominal matrix $\mathbf{A}(\lambda)$ is assumed to be $\partial \mathcal{D}$-regular with $\partial \mathcal{D}$ as defined by (9) with (6)-(8) and (10). The purpose is here to derive what can be called the complex $\partial \mathcal{D}$-regularity radius $\varrho_{\partial \mathcal{D}}$, which is the largest value of the radius $\rho$ such that the uncertain polynomial matrix $\mathbb{A}(\lambda)$ defined by (44) remains $\partial \mathcal{D}$-regular over $\mathcal{B}(\rho)$. To reach such a goal, $\Theta$ is chosen as follows:

$$
\Theta:=\left[\begin{array}{ll}
C^{\prime} C & C^{\prime} D \\
D^{\prime} C & D^{\prime} D-\gamma I
\end{array}\right], \quad \gamma=\rho^{-1 / 2} .
$$

Corollary 1 Let an uncertain $k$-variate matrix $\mathbb{A}(\lambda)$ be defined as in (44) and a set $\partial \mathcal{D}$ be defined by (9) with (6)-(8) and (10). $\mathbb{A}(\lambda)$ is robustly $\partial \mathcal{D}$-regular against $\mathcal{B}(\rho)$ if there exists a set $\mathcal{P} \in \mathbb{H}$ such that (25) holds with $M$ defined as in (21), $\overline{\mathbf{R}}(\mathcal{P})$ defined by (15), $F$ given by (43) and $\Theta$ given by (47).

Proof It consists in applying Theorem 1. With the choice (47), the property (i) in Theorem 1 can be expressed

$$
\|G(\lambda)\|_{2}<\sqrt{\gamma} \quad \forall \lambda \in \partial \mathcal{D}
$$

(with $G(\lambda)$ given by (2)), by noting that the choice of $F$ proposed in (43) leads to

$$
\operatorname{Span}(N(\lambda))=\operatorname{Span}\left(\left[\begin{array}{c}
(E H(\lambda)-A)^{-1} B \\
I
\end{array}\right]\right),
$$


since $E$ is blockdiagonal. Then, inequality (48) can be written as

$$
\left\{\sup _{\lambda \in \partial \mathcal{D}} \bar{\sigma}(G(\lambda))\right\}^{-1}>\rho .
$$

Simple arguments on singular values show that

$$
\bar{\sigma}(G(\lambda))=\mu_{\mathbb{C}}(G(\lambda))
$$

where $\mu_{\mathbb{C}}$ (.) denotes the celebrated complex structured singular value. Then it comes

$$
\left\{\sup _{\lambda \in \partial \mathcal{D}} \mu_{\mathbb{C}}(G(\lambda))\right\}^{-1}>\rho .
$$

Taking the fact that

$$
\mu_{\mathbb{C}}(G(\lambda))=\left[\inf _{\Delta}\{\bar{\sigma}(\Delta): \operatorname{det}(I-G(\lambda) \Delta)=0\}\right]^{-1}
$$

into account, one gets

$$
\inf _{\lambda \in \partial \mathcal{D}}\left\{\inf _{\Delta}\{\bar{\sigma}(\Delta): \operatorname{det}(I-G(\lambda) \Delta)=0\}\right\}>\rho .
$$

Besides,

$$
\begin{aligned}
& \operatorname{det}(I-G(\lambda) \Delta)=0 \\
& \Leftrightarrow \operatorname{det}\left(I-C(E H(\lambda)-A)^{-1} B \Delta-D \Delta\right)=0 \\
& \Leftrightarrow \operatorname{det}\left(I-C(E H(\lambda)-A)^{-1} B \Delta\left(I_{p}-D \Delta\right)^{-1}\right) \operatorname{det}\left(I_{p}-D \Delta\right)=0 .
\end{aligned}
$$

From the well posedness assumption, $\operatorname{det}\left(I_{p}-D \Delta\right) \neq 0$, the above equality is equivalent to

$$
\begin{aligned}
& \operatorname{det}\left(I-C(E H(\lambda)-A)^{-1} B \bar{\Delta}\right)=0 \\
& \Leftrightarrow \operatorname{det}\left(I-(E H(\lambda)-A)^{-1} B \bar{\Delta} C\right)=0 \\
& \Leftrightarrow \operatorname{det}\left((E H(\lambda)-A)^{-1}\right) \operatorname{det}(E H(\lambda)-A-B \bar{\Delta} C)=0 .
\end{aligned}
$$

Since, for $\Delta=0, \mathbb{A}(\lambda)=\mathbf{A}(\lambda)$ is implicitly assumed to be $\partial \mathcal{D}$-regular (otherwise why testing robust $\partial \mathcal{D}$-regularity?), the left factor of the left handside member is non zero so it follows that

$$
\operatorname{det}(E H(\lambda)-A-\bar{A})=0 .
$$

From (54) and (55), it can be deduced that

$$
\inf _{\lambda \in \mathcal{D}}\left\{\inf _{\Delta}\{\bar{\sigma}(\Delta): \operatorname{det}(\mathbb{A}(\lambda))=0\}\right\}>\rho,
$$

which proves that $\mathbb{A}(\lambda)$ remains $\partial \mathcal{D}$-regular against $\mathcal{B}(\rho)$.

It is clear that $\rho^{\star}$, the maximum value of $\rho=\gamma^{-1 / 2}$ obtained by computing $\mathcal{P}$ proving (24), is a lower bound of $\varrho_{\partial \mathcal{D}}$. If $k=1$, then $\rho^{\star}=\varrho_{\partial \mathcal{D}}$. Note, however, that matrix $\Delta$ is here assumed to be complex. In practice, it would be interesting to take its possible realness into account.

In the remaining part of the article, it will be assumed that

$$
E=I \Rightarrow F=\left[\begin{array}{cc}
A & B \\
I & 0
\end{array}\right],
$$

in order to apply this quite general corollary to the analysis of the actual model (1). 


\subsubsection{Application to asymptotic stability analysis of hybrid Roesser model}

In this part, the model (1) is considered in association with the uncertainty structure defined by the feedback equation

$$
u\left(t_{1}, \ldots, t_{r}, j_{r+1}, \ldots, j_{k}\right)=\Delta y\left(t_{1}, \ldots, t_{r}, j_{r+1}, \ldots, j_{k}\right), \Delta \in \mathcal{B}(\rho) \cap \mathbb{R}^{m \times p},
$$

The above equation corresponds to the LFT formalism mentioned in the previous subsection.

Application of (58)-(1) yields the closed-loop state matrix

$$
A_{c}=A+\bar{A},
$$

where $\bar{A}$ is given by (45)-(46).

Before to study various performance levels in the next subsection, the main property is considered, namely asymptotic stability. Applying Corollary 1 with $E=I$ enables ones to test, with conservatism, if matrix $\mathbb{A}(\lambda)=H(\lambda)-A_{c}$ is robustly $\partial \mathcal{D}$-regular against $\mathcal{B}(\rho)$. As mentioned at the end of subsection 2.3, asymptotic stability of Roesser model (1) depends on the root-clustering of polynomial $c(\lambda, A)$. Thus, robust stability of the uncertain hybrid Roesser model described by (1) and (58) against $\mathcal{B}(\rho)$ depends on the root-clustering of $c\left(\lambda, A_{c}\right)=\operatorname{det}(\mathbb{A}(\lambda))$. More precisely, for $c\left(\lambda, A_{c}\right)$ to match the robust stability conditions induced by Lemma 1 , it is necessary that $\mathbb{A}(\lambda)$ be at least $\partial \mathcal{D}$-regular with the choice (10). Following this idea, the next theorem is stated

Theorem 2 Suppose that a multidimensional hybrid system of the form described by (1) is subject to uncertainty (58). Then the resulting uncertain system is robustly asymptotically stable against $\mathcal{B}(\rho)$ if there exists a set $\mathcal{P}$ of $k$ matrices $P_{i} \in \mathcal{H}_{n_{i}}^{+}, i=1, \ldots, k$, such that the following LMI holds

$$
F^{\prime} \overline{\mathbf{R}}(\mathcal{P}) F+\Theta<0
$$

where $F$ is given by (57), $\overline{\mathbf{R}}(\mathcal{P})$ is given by (15) with (10), and $\Theta$ is given by (47).

Proof Based on Corollary 1, we conclude that the LMI (60) implies that $\mathbb{A}(\lambda)$ is robustly $\partial \mathcal{D}$-regular against $\mathcal{B}(\rho)$ with $\partial \mathcal{D}$ characterized by (10). But it is not just another corollary. Since the matrices $P_{i}$ are assumed to be positive definite, then the first block in (60), which is

$$
\left[\begin{array}{c}
A \\
I
\end{array}\right]^{\prime} \overline{\mathbf{R}}(\mathcal{P})\left[\begin{array}{c}
A \\
I
\end{array}\right]+C^{\prime} C<0 \Rightarrow\left[\begin{array}{c}
A \\
I
\end{array}\right]^{\prime} \overline{\mathbf{R}}(\mathcal{P})\left[\begin{array}{c}
A \\
I
\end{array}\right]<0,
$$

proves asymptotic stability of the nominal system (1). To see this, note that the columns of

$$
\left[\begin{array}{l}
A \\
I_{n}
\end{array}\right]
$$

span the kernel of

$$
\left[I_{n}-A\right]
$$

and, in the sequel, invoke the matrix elimination procedure (Boyd et al. 1994) to observe that there exists a matrix $G=G^{\prime}$ such that

$$
\mathbf{N}=\overline{\mathbf{R}}(\mathcal{P})+\left[\begin{array}{c}
I_{n} \\
-A^{\prime}
\end{array}\right] G\left[I_{n}-A\right]<0 .
$$


Further, let a vector $\lambda$ (as introduced in (11)) comply with $c(\lambda, A)=0$. It will be proven that $\lambda$ cannot lie in $\mathcal{S}^{C}$ defined as in (5). Indeed, if $c(\lambda, A)=0$, there exists a vector $v \in \mathbb{C}^{n}$ such that

$$
\mathbf{A}(\lambda) v=0
$$

Let the vector $q$ be defined by

$$
q=\left[\begin{array}{c}
H(\lambda) \\
I_{n}
\end{array}\right] v
$$

Note that, if $v$ is written

$$
v=\left[\begin{array}{c}
v_{1} \\
\vdots \\
v_{k}
\end{array}\right], \quad \text { with } \quad v_{i} \in \mathbb{C}^{n_{i}} \forall i \in \mathbb{I}(k),
$$

then $q$ becomes

$$
q=\left[\begin{array}{c}
\lambda_{1} v_{1} \\
\vdots \\
\lambda_{k} v_{k} \\
v_{1} \\
\vdots \\
v_{k}
\end{array}\right] .
$$

Now, based on (62), it can be deduced that

$$
q^{\prime} \mathbf{N} q<0
$$

which can be rewritten as

$$
\sum_{i=1}^{k}\left(v_{i}^{\prime} P_{i} v_{i} \mathcal{F}_{R_{i}}\left(\lambda_{i}\right)\right)+v^{\prime} \mathbf{A}^{\prime}(\lambda) G \mathbf{A}(\lambda) v<0 .
$$

From (63), it can be seen that the second term in the above inequality is zero. Moreover, the set $\mathcal{S}^{C}$ can be defined (with the choice (10)), by $\mathcal{F}_{R_{i}} \geq 0$. Thus, if $\lambda$ belongs to $\mathcal{S}^{C}$, then the first term in (64) is positive or zero, which contradicts (64). Hence, $\lambda$ cannot belong to $\mathcal{S}^{C}$. Then $c(\lambda, A)=\operatorname{det}(\mathbf{A}(\lambda))$ satisfies the condition of Lemma 1, implying nominal stability. It is clear that $c\left(\lambda, A_{c}\right)=\operatorname{det}(\mathbb{A}(\lambda))$ also satisfies the condition of Lemma 1 for any $\Delta$ otherwise, by continuity of $\lambda$ with respect to $\Delta, \mathbb{A}(\lambda)$ would becomes $\partial \mathcal{D}$-singular. Hence, (1) with (58) is robustly asymptotically stable against $\mathcal{B}(\rho)$.

It can be noted again that the maximum value of $\rho$ while satisfying LMI (60), denoted by $\rho^{\star}$, is a lower bound of the complex stability radius.

The strong difference between $\partial \mathcal{D}$-regularity and asymptotic stability lies in the distribution of the roots of $c\left(\lambda, A_{c}\right)$ with respect to $\partial \mathcal{D}$. Basically, Theorem 2 ensures robust $\partial \mathcal{D}$ regularity. However, if nominal $k$-variate polynomial matrix is "stable", meaning that $c(\lambda, A)$ fills the conditions given by Lemma 1 or equivalently that (1) is asymptotically stable, then this property is obviously preserved when $\Delta$ describes $\mathcal{B}(\rho)$ if $\mathbb{A}(\lambda)$ remains $\partial \mathcal{D}$-regular. Nevertheless, if, for some $\Delta$, matrix $\mathbb{A}(\lambda)$ becomes $\partial \mathcal{D}$-singular, it does not necessarily imply instability. Thus, the conservatism of Theorem 2 is due both to this reason and to the fact that 
Corollary 1 also proposes a sufficient condition. Another reason is that the realness of $\Delta$ is not taken into account.

In the 1-D case, only this last reason induces conservatism. Indeed, in this case, not only Theorem 1 and thus Corollary 1 provide a necessary and sufficient condition but moreover, $\partial \mathcal{D}$-singularity implies instability.

\subsection{Positive realness of hybrid Roesser model}

Since the work of Popov on hyperstability, positive realness (Anderson 1968) of transfer matrices have been widely investigated, especially for classical 1-D systems, noting that, for such models, positive realness means passivity and enables the designer to include sectorbounded nonlinearities. For multidimensional linear models, few contributions exist. However, the 2-D discrete Roesser models are considered from the viewpoint of positive realness in $\mathrm{Xu}$ et al. (2003). Hence, we make an attempt to extend those results to investigate positive realness of hybrid Roesser models.

We will first state the following definition.

Definition 2 Let the set $\partial \mathcal{D}$ be defined by (9) with (6)-(8) and (10). The model (1) is said to be strictly positive real over $\partial \mathcal{D}$ if

$$
G(\lambda)+G^{\prime}(\lambda)>0 \quad \forall \lambda \in \partial \mathcal{D} .
$$

Following this idea, the next corollary is proposed.

Corollary 2 Consider a hybrid Roesser model and the set $\partial \mathcal{D}$ be described by (1) and (9) with (6)-(8) and (10) respectively. Suppose also that $M$ is defined in (21), $\overline{\mathbf{R}}(\mathcal{P})$ is defined by (15), $F$ is given by (57) and

$$
\Theta:=\left[\begin{array}{ll}
0 & -C^{\prime} \\
-C & -D-D^{\prime}
\end{array}\right] .
$$

Then, the model (1) is strictly positive real over $\partial \mathcal{D}$ if there exists a set $\mathcal{P} \in \mathbb{H}$ such that (25) holds.

Proof It follows immediately form Theorem 1 that with (66), the expression 23 corresponds to $(65)$.

It is clear that the notion of strict positive realness over $\partial \mathcal{D}$ might not be appropriate to appreciate the performances of a $n \mathrm{D}$-model and one can come back to the more particular but classical notion of strict positive realness.

Definition 3 The model (1) is strictly positive real if $G(\lambda)$ is analytic on $\mathcal{S}^{C}$ defined by (5) and if inequality

$$
G(\lambda)+G^{\prime}(\lambda)>0
$$

hold on $\mathcal{S}^{C}$.

Theorem 3 Consider a multidimensional hybrid system described by Roesser model (1). Suppose also that $F$ is given by $(57), \overline{\mathbf{R}}(\mathcal{P})$ is given by (15) with (10) and $\Theta$ is given by (66). Then, the hybrid $n-D$ system is strictly positive real if there exists a set $\mathcal{P}$ of $k$ matrices $P_{i} \in \mathcal{H}_{n_{i}}^{+}, i=1, \ldots, k$, such that LMI (60) holds.

Proof This follows immediately from the result of Corollary 2 except that the matrices $P_{i}$ are assumed to be positive definite in order to guarantee asymptotic stability as in the proof of Theorem 2 . 
It is significant to note that Theorem 3 can be seen as an extension of the LMI version of the classical positive real lemma (see Boyd et al. 1994 for example).

\subsection{Bounded realness of hybrid Roesser model}

The idea is about the same as in the above subsection but the performance to be considered is the so-called $\mathcal{H}_{\infty}$-level. As some extension of the $\mathcal{H}_{\infty}$-norm proposed in Du and Xie (2002) for the 2-D discrete Roesser models, the following definition introduces the $\mathcal{L}_{\infty}$-norm of model (1) with respect to $\partial \mathcal{D}$.

Definition 4 Let a hybrid Roesser model and the set $\partial \mathcal{D}$ be described by (1) and (9) with (6)-(8) and (10) respectively. Then, the $\mathcal{L}_{\infty}$-norm of (1) with respect to $\partial \mathcal{D}$ is defined by

$$
\|G\|_{\infty}=\sup _{\lambda \in \partial \mathcal{D}}\|G(\lambda)\|_{2}
$$

From this definition and from Theorem 1, the next corollary is stated.

Corollary 3 Let the hybrid Roesser model and the set $\partial \mathcal{D}$ be described by (1) and (9) with (6)-(8) and (10) respectively. The $\mathcal{L}_{\infty}$-norm of (1) with respect to $\partial \mathcal{D}$ is strictly lower than $\sqrt{\gamma}$ if there exists a set $\mathcal{P} \in \mathbb{H}$ such that (25) holds with $M$ defined as in (21), $\overline{\mathbf{R}}(\mathcal{P})$ defined by (15), $F$ given by (57) and $\Theta$ given by (47).

Proof Direct from the discussion in subsection 4.1.1 and especially inequality (48).

Assuming that (1) is asymptotically stable (i.e. $G(\lambda)$ is analytic on $\mathcal{S}^{C}$ ), then the $\mathcal{L}_{\infty}$-norm becomes the so-called $\mathcal{H}_{\infty}$-norm.

Definition 5 Let the hybrid Roesser model (1) comply with Lemma 1 . Its $\mathcal{H}_{\infty}$-norm is defined by (68) with (9) and (6)-(8) together with (10).

This $\mathcal{H}_{\infty}$-norm actually equals the so-called $\mathcal{L}_{2}$-gain but this connection is not detailed here for the sake of conciseness.

From the above definition, the next corollary is formulated.

Theorem 4 Let the hybrid Roesser model be described by (1). It is asymptotically stable and its $\mathcal{H}_{\infty}$-norm is strictly lower than $\sqrt{\gamma}$ if there exists a set $\mathcal{P}$ of $k$ matrices $P_{i} \in \mathcal{H}_{n_{i}}^{+}$, $i=1, \ldots, k$, such that LMI (60) holds, where $F$ is given by $(57), \overline{\mathbf{R}}(\mathcal{P})$ is given by (15) with (10) and $\Theta$ is given by (47).

Proof The proof follows immediately from the result of Corollary 3 combined with (10) and under assumption that the matrices $P_{i}$ are positive definite to ensure asymptotic stability.

Theorem 4 can be seen as an extension of the classical LMI version of the Bounded real lemma (Gahinet and Apkarian 1994) to hybrid Roesser models. It has to be mentioned that the $\mathcal{H}_{\infty}$-control design is considered in Xu et al. (2005) through an equivalent approach.

Remark 1 Corollaries 1 and 3 actually involve the same conditions. The same for Theorems 2 and 4 . In Corollary 1 (resp. Theorem 2 ), robust $\partial \mathcal{D}$-regularity (resp. robust stability) is concerned whereas in Corollary 3 (resp. Theorem 4 ), $\mathcal{L}_{\infty}$-norm on $\partial \mathcal{D}$ (resp. $\mathcal{H}_{\infty}$ performance level) is addressed. This connection between the $\mathcal{L}_{\infty}$ or $\mathcal{H}_{\infty}$-norm of a system and the quadratic stability against an LFT-based uncertainty is already well known in the 1-D system case (Khargonekar et al. 1990). 


\section{Numerical example}

In this section, the results developed above are illustrated by one numerical example.

One focuses on the computation of a minimum upper bound of the $\mathcal{H}_{\infty}$ norm of a model describing the behavior of a metal rolling process. This can be done by using control theory for differential linear repetitive processes. To proceed, a differential linear repetitive statespace model for that metal rolling process has to be developed. This model is obtained in, for example, Rogers et al. (2007), where the resulting state-space equations involve the following matrices:

$$
\begin{aligned}
& \mathcal{A}=\left[\begin{array}{ll}
-0.0050 & -5.8077 \\
1 & -0.0050
\end{array}\right], \mathcal{B}_{0}=\left[\begin{array}{l}
0 \\
0.0494
\end{array}\right], \\
& \mathcal{C}=\left[\begin{array}{ll}
1 & 0
\end{array}\right], \mathcal{D}_{0}=0.7692, \mathcal{B}_{1}=\left[\begin{array}{l}
0.9 \\
0.2
\end{array}\right], \mathcal{D}_{1}=0.6
\end{aligned}
$$

(see Rogers et al. 2007 for the details, especially the meaning of the above matrices). It turns out that the above process is not stable. Application of a static controller design procedure (which it is no use to detailing here) results in a stable closed-loop differential linear repetitive process the state-space model of which is associated to the next matrices:

$$
\begin{aligned}
& \mathcal{A}^{c l}=\left[\begin{array}{ll}
-0.3650 & -10.3077 \\
0.9200 & -1.0050
\end{array}\right], \mathcal{B}_{0}^{c l}=\left[\begin{array}{l}
-0.2700 \\
-0.0106
\end{array}\right], \\
& \mathcal{C}^{c l}=\left[\begin{array}{ll}
0.7600 & -3.0000
\end{array}\right], \mathcal{D}_{0}^{c l}=0.5892 .
\end{aligned}
$$

What is important here is that, based on transformations shown in Rogers et al. (2007), the above model takes the hybrid 2-D state-space model structure (1) with $r=2$ (it means that the system has continuous dynamics along two of three dimensions) and the associated matrices are given by

$$
\begin{aligned}
& A=\left[\begin{array}{ll}
\mathcal{A}^{c l} & \mathcal{B}_{0}^{c l} \\
\mathcal{C}^{c l} & \mathcal{D}_{0}^{c l}
\end{array}\right], \quad B=\left[\begin{array}{l}
\mathcal{B}_{1} \\
\mathcal{D}_{1}
\end{array}\right] \\
& C=\left[\begin{array}{lll}
0 & 0 & 1
\end{array}\right], \quad D=0
\end{aligned}
$$

Now, the result of Theorem 4 allows us to compute the minimum upper bound on $\mathcal{H}_{\infty}$ norm of the system described by the above matrices.

With computations performed owing to LMI CONTROL TOOLBOX (Gahinet et al. 1995), it is verified that this system is asymptotically stable and the minimum $\mathcal{H}_{\infty}$ performance level $\rho=\sqrt{\gamma}$ is 2.0790. Furthermore, the matrices that solve the LMI (60) are

$$
P_{1}=\left[\begin{array}{ll}
6.6991 & -4.1148 \\
-4.1148 & 67.1344
\end{array}\right], \quad P_{2}=2.0349
$$

Indeed, in this case, $P_{2}$ is a scalar. Following Remark 1 , it also means that $\rho$ is a lower bound of the complex stability radius. 


\section{Conclusion}

\subsection{Summary}

In this paper, a KYP-like theorem has been proposed with a purpose of application to the very general hybrid state-space Roesser models. As a special instance of the result, the KYP lemma for classical 1-D linear models is recovered. Unlike for this 1-D case, for multidimensional models, some conservatism appears. This is due to the nature of Roesser models themselves. The origin of this conservatism has been highlighted.

Some possibilities of exploiting this new theorem in terms of robust stability, positive realness and $\mathcal{H}_{\infty}$-analysis of hybrid Roesser models have been emphasized. The authors hope that this point of view will be seen as an attempt to unify numerous contributions dealing with LMI approach applied to $n$-D models. But they also hope that it will arouse many other contributions relevant to $n$-D KYP lemma through alternative approaches. In that sense, the next discussion aims at proposing possible tracks for further investigations.

\subsection{Discussion about perspectives}

The present work is clearly a contribution to the analysis of $n$-D models. No design technique is proposed. However, the authors would like to stress the link between some existing synthesis LMI conditions, such as those in Gałkowski et al. (2003a,b,c), Xu et al. (2003) for example, and the framework presented here. Actually, control conditions provided in the above references are based upon analytical conditions that can be subsumed to the present version of $n$-D KYP lemma. For this reason, the authors think that Theorem 1 is more than likely to generate design conditions, possibly (or probably) with additional conservatism, especially with state feedback controller or maybe dynamic output feedback controller with the same order as the plant. For static output feedback design or reduced order dynamic output feedback design, the exploitation of condition (ii) in Theorem 1 is a far more difficult challenge, keeping in mind that even for the 1-D case, this kind of problem is still widely open. So, clearly, this approach, though here presented in an analytical context, can be seen as a starting point to address design problems.

In the present contribution, Theorem 1 was proved owing to the well known S-procedure, using the formalism proposed in Iwasaki and Hara (2005). Exploiting the S-procedure to derive KYP-like conditions has now become rather traditional. Indeed, the kinship between the KYP lemma and the S-procedure is nearly historical and was very well highlighted in Gusev and Likhtarnikov (2006). Thus, the authors of the present article were quite naturally led to follow this traditional way to solve their problem. It has the advantage to introduce conditions in which "Lyapunov matrices" $P_{i}$ (in a very large sense) are involved, following the existing background on LMI conditions for analysis and control of $n$-D models. However, there are other approaches to the derivation of KYP-like inequalities, such as dissipative systems (Willems 1972a,b), Integral quadratic constraints (IQC) (Megretski and Rantzer 1997), well-posedness of feedback systems (Safonov 1980), and so on... Actually, the problem considered in the present paper is to test condition (i) in Theorem 1 (with the constraint $\lambda \in \partial \mathcal{D}$ ) which equivalently consists in testing the nonnegativity a polynomial matrix over $\partial \mathcal{D}$. Theorem 1 (here referred to as the $n$-D KYP lemma) can be seen as a way to transform the nonnegativity of this $n$-D polynomial matrix into a matrix inequality problem (preferably an LMI), i.e. a condition which is numerically tractable. The great success of the KYP lemma lies in the fact that this relaxation, in the 1-D case, induces no conservatism. The lossless S-procedure simply appears a possible means to achieve this relaxation. Thanks to 
an anonymous reviewer, the authors are now aware that it is far from being the only one. For instance, if one considers the problem of testing condition (i) in Theorem 1 with the lens of optimization theory, some relaxations could be found by using Lagrange duality theory. 1-D KYP lemma can be subsumed to this theory (see Scherer 2006 and the references therein) and in that case, the duality gap can be proved to vanish. More generally ( $n$-D case for example), the problem would be to find relaxations of the original condition for which the duality gap is weak and, if possible, vanishes.

Among the possible relaxations are the so-called Sum-Of-Squares (SOS)-descriptions. More precisely, it is known that testing the nonnegativity of a multivariate polynomial is in general NP-hard and a sufficient condition is that this polynomial has a SOS-description. Besides, the problem of the existence of a SOS-description can be converted into a semidefinite program of possibly high dimension (Lasserre 2001; Parrilo 2003). So, asserting the feasibility of such a program is a way to guarantee the nonnegativity of a polynomial. The Sprocedure can serve the same purpose. Actually, the strong link between SOS formulation and S-procedure was well highlighted in de Oliveira (2005). In the same fashion, 1-D KYP lemma can be seen as the equivalence between nonnegativity of a univariate polynomial matrix (over a curve $\partial \mathcal{D}$ ) and an LMI corresponding to the existence of a SOS-formulation of this matrix (Genin et al. 2002, 2003). The expression in the condition (i) of Theorem 1 involves an $n$-variate polynomial matrix (with variables in a specified region). Again, a sufficient condition for such a matrix to be nonnegative is the existence of a SOS-description. With the recent extensions of Hol and Scherer (2004, 2005), Kojima (2003), this latter test could be relaxed under an LMI problem the dimension of which might be high to guarantee the non conservatism of the relaxation. Moreover, a recent paper (Henrion and Lasserre 2006) showed the interest in using the moment interpretation of matrix SOS-decomposition not only to reduce the size of the LMI relaxation but also to extract the optimizers. Therefore, not only the LMI problem to be solved is of lower dimension but also, the possibility to extract optimizers could reveal as very useful in a design context to derive the controller. Indeed, the authors of Henrion and Lasserre (2006) illustrated the relevance of their approach, in the 1-D case, to solve problems which are basically associated with the crucial static output feedback control. Consequently, their work might be a starting point for the design of control laws for $n$-D models.

At last, another topic which is adressed in the present article is the robust stability of Roesser models. Theorem 2 gives a sufficient condition for model (1) with $E=I$ to be robustly asymptotically stable against an LFT uncertainty. This condition ensures that the characteristic polynomial $c\left(\lambda, A_{c}\right)$ matches the stability conditions given in Lemma 1 . It is important to notice that these stability conditions, in the purely continuous case, correspond to what is referred to as strict sense stability of a $n$-variate polynomial in Kharitonov and Torres Muñoz (1999). In this reference, Kharitonov and Torres Muñoz explain that this property is not sufficient enough in the robust context since some very small deflection of one coefficient might not preserve strict sense stability. This is why they propose a new kind of stability (see Kharitonov and Torres Muñoz 1999, Theorem 13). It means that, if condition (60) is satisfied with $E=I$ and $r=k$, then (1) is robustly strict sense stable against (59) with (45)-(46) but any other uncertainty on the Roesser model, not included in this LFT-structure, might induce a destabilizing deflection in the coefficients of the characteristic polynomial. In a practical context, it might be reasonable to take this fragility into account. Actually, it is quite easy to see that part of the properties required for a polynomial to be stable in the sense of Kharitonov and Torres Muñoz (1999, Theorem 13) are verified and it would be interesting to work on that topic in order to (perhaps?) prove that the uncertain characteristic polynomial, with its particular structure, is stable in the sense of Kharitonov and Torres Muñoz (1999, Theorem 13) when it is strict sense stable. 
The authors confess that they are not familiar with all the technical details that would arise from the suggested perspectives but they hope that this discussion will be fruitful for the study of $n$-D systems.

Open Access This article is distributed under the terms of the Creative Commons Attribution Noncommercial License which permits any noncommercial use, distribution, and reproduction in any medium, provided the original author(s) and source are credited.

\section{References}

Agathoklis, P. (1988). Lower bounds for the stability margin of discrete two-dimensional systems based on the two-dimensional Lyapunov equation. IEEE Transactions on Circuits and Systems, 35(6), 745-749.

Anderson, B. D. O. (1968). A simplified viewpoint on hyperstability. IEEE Transactions on Automatic Control, 13(3), 292-294.

Bachelier, O., Henrion, D., Pradin, B., \& Mehdi, D. (2004). Robust matrix root-clustering of a matrix in intersections or unions of subregions. SIAM Journal of Control and Optimization, 43(3), 1078-1093.

Basu, S. (2002). Multidimensional causal, stable, perfect reconstruction filter banks. IEEE Transactions on Circuits and Systems I: Fundamental Theory and Applications, 49(6), 832-842.

Bochniak, J., \& Gałkowski, K. (2005). LMI-based analysis for continuous-discrete linear shift invariant nDsystems. Journal of Circuits, Systems and Computers, 14(2), 1-26.

Bose, N. K. (1982). Applied multidimensional systems theory. New York, USA: Van Nostrand-Reinhold.

Bose, N. K. (1985). Multidimensional systems theory. Progress, directions and open problems in multidimensional systems. Dordrecht, Holland: D. Reidel Publishing Company.

Boyd, S., Ghaoui, L. E., Feron, E., \& Balakrishnan, V. (1994). Linear matrix inequalities in system and control theory, volume 15 of SIAM studies in applied and numerical mathematics. SIAM, Philadelphia, USA.

Bracewell, R. N. (1995). Two-dimensional imaging. Upper Saddle River, USA: Prentice Hall Signal Processing Series. Prentice Hall Inc.

de Oliveira, M. C. (2005). Decomposition of a polynomial as a Sum-Of-Squares of polynomials and the S-procedure. In Proc. 44th IEEE Conf. Decision and Control (CDC) and the European Control Conference (ECC) 2005 (pp. 1654-1659). Seville, Spain, 12-15 December 2005.

Du, C., \& Xie, L. (2002). $\mathcal{H}_{\infty}$ Control and Filtering of two-dimensional systems, volume 278 of lecture notes in control and information sciences. Berlin, Germany: Springer-Verlag.

Dudgeon, D. E., \& Merserau, R. M. (1984). Multidimensional digital signal processing. Englewood Cliffs, USA: Prentice-Hall Signal Processing Series. Prentice Hall.

Ebihara, Y., Ito, Y., \& Hagiwara, T. (2006). Exact stability analysis of 2-D systems using LMIs. IEEE Transactions on Automatic Control, 51(9), 1509-1513.

Fornasini, E., \& Marchesini, G. (1978). Doubly indexed dynamical systems: State models and structural properties. Mathematical Systems Theory, 12, 59-72.

Fu, P., Chen, J., \& Niculescu, S.-I. (2006). Generalized eigenvalue-based stability tests for 2-D linear systems: Necessary and sufficient conditions. Automatica, 42, 1569-1576.

Gahinet, P., \& Apkarian, P. (1994). A linear matrix inequality approach to $\mathcal{H}_{\infty}$ control. International Journal of Robust and Nonlinear Control, 4, 421-448.

Gahinet, P., Nemirovski, A., Laub, A. J., \& Chilali, M. (1995). LMI control toolbox for use with MATLAB. Natick, USA: The Mathworks Partner Series. The MathWorks Inc.

Gałkowski, K., \& Wood, J. (Eds.). (2001). Multidimensional signals, circuits and systems. London, England: Systems and Control Book Series. Taylor and Francis.

Gałkowski, K., Lam, J., Rogers, E., Xu, S., Sulikowski, B., Paszke, W., \& Owens, D. H. (2003a). LMI based stability analysis and robust controller design for discrete linear repetitive processes. International Journal of Robust and Nonlinear Control, 13(13), 1195-1211.

Gałkowski, K., Lam, J., Xu, S., \& Lin, Z. (2003b). LMI approach to state-feedback stabilization of multidimensional systems. International Journal of Control, 76(14), 1428-1436.

Gałkowski, K., Paszke, W., Rogers, E., Xu, S., Lam, J., \& Owens, D. H. (2003c). Stability and control of differential linear repetitive processes using an LMI setting. IEEE Transactions on Circuits and Systems-II: Analog and Digital Signal Processing, 50(9), 662-666.

Genin, Y., Hachez, Y., Nesterov, Y., Stefan, R., Van Dooren, P., \& Xu, S. (2002). Positivity and linear matrix inequalities. European Journal of Control, 8(3), 275-298. 
Genin, Y., Hachez, Y., Nesterov, Y., \& Van Dooren, P. (2003). Optimization problems over positive pseudopolynomial matrices. SIAM Journal on Matrix Analysis and Applications, 25(1), 57-79.

Gusev, S. V., \& Likhtarnikov, A. L. (2006). Kalman-Popov-Yakubovich lemma and the S-procedure: A historical essay. Automation and Remote Control, 67(11), 1768-1810.

Henrion, D., \& Lasserre, J.-B. (2006). Convergent relaxations of polynomial matrix inequalities and static ouput feedback. IEEE Transactions on Automatic Control, 51(2), 192-202.

Henrion, D., Šebek, M., \& Bachelier, O. (2001). Rank-one LMI approach to stability of 2-D polynomial matrices. Multidimensional Systems and Signal Processing, 12(1), 33-48.

Hinamoto, T. (1993). 2-D Lyapunov equation and filter design based on the Fornasini-Marchesini second model. IEEE Transactions on Circuits and Systems I: Fundamental Theory and Applications, 40(2), 102110.

Hol, C. W., \& Scherer, C. W. (2004). Sum of squares relaxations for polynomial semidefinite programming. In Proc. Symp. on Mathematical Theory of Networks and Systems (MNTS), Leuven, Belgium, July 2004.

Hol, C. W., \& Scherer, C. W. (2005). A sum of squares approach to fixed-order $\mathrm{H}_{\infty}$ synthesis, Contributed chapter. In D. Henrion \& A. Garulli (Eds.), Positive polynomials in control, Signal Processing, Optimization and Control. LNCIS Springer Verlag, Berlin.

Iwasaki, T., \& Hara, S. (2005). Generalized KYP lemma: Unified frequency domain inequalities with design applications. IEEE Transactions on Automatic Control, 50(1), 41-59.

Jury, E. (1978). Stability of multidimensional scalar and matrix polynomial. Proceedings of the IEEE, 66(9), 1018-1047.

Kaczorek, T. (1985). Two-dimensional linear systems, volume 68 of lecture notes in control and information sciences. Springer-Verlag, Berlin, Germany.

Khargonekar, P. P., Petersen, I. R., \& Zhou, K. (1990). Robust stabilization of uncertain linear systems: Quadratic stabilizability and $\mathcal{H}_{\infty}$ control theory. IEEE Transactions on Automatic Control, 35(3), 356-361.

Kharitonov, V. L., \& Torres Muñoz, J. A. (1999). Robust stability of multivariate polynomials. Multidimensional Systems and Signal Processing, 10, 7-20.

Kojima, M. (2003). Sum of squares relaxations of semidefinite programs. Research report B-397, Dept. Math. Comp. Sc., Tokyo Inst. Tech., Japan, November 2003.

Lasserre, J.-B. (2001). Global optimization with polynomials and the problem of moments. SIAM Journal on Optimization, 11(3), 796-817.

Lu, W.-S. (1994). On a Lyapunov approach to stability analysis of 2-D digital filters. IEEE Transactions on Circuits and Systems I: Fundamental Theory and Applications, 41(10), 665-669.

Lu, W.-S., \& Antoniou, A. (1992). Two-dimensional digital filters, volume 80 of electrical engineering and elecronics. Marcel Dekker, Inc., New York, USA.

Megretski, A., \& Rantzer, A. (1997). System analysis via Integral Quadratic Constraints. IEEE Transactions on Automatic Control, 42, 819-830.

Parrilo, P. A. (2003). Semidefinite programming relaxations for semialgebric problems. Mathematical Programming, 96(2), 293-320.

Piekarski, M. (1977). Algebraic characterization of matrices whose multivariable characteristic polynomial is Hurwitzian. In Proc. Int. Symp. Operator Theory. Lubbock, USA.

Rantzer, A. (1996). On the Kalman-Yakubovich-Popov lemma. Systems and Control Letters, 28(1), 7-10.

Roesser, R. P. (1975). A discrete state-space model for linear image processing. IEEE Transactions on Automatic Control, 20(1), 1-10.

Rogers, E., Gałkowski, K., \& Owens, D. H. (2007). Control systems theory and applications for linear repetitive processes, volume 349 of lecture notes in control and information sciences. Springer-Verlag, Berlin, Germany.

Safonov, M. G. (1980). Stability and robustness of multivaribale feedback systems. Signal Processing, Optimization and Control. Cambridge, MA: MIT Press.

Scherer, C. W. (2006). LMI relaxations in robust control. To appear in European Journal of Control.

Šebek, M. (1988). n-D matrix polynomial equations. IEEE Transactions on Automatic Control, 33(5), 499502.

Shi, Y. Q., \& Zhang, X. M. (2002). A new two-dimensional interleaving technique using successive packing. IEEE Transactions on Circuits and Systems I: Fundamental Theory and Applications, 49(6), 779-789.

Willems, J. C. (1972a). Dissipative dynamical, part I: General theory. Arch. Ratinal Mech. Anal, 45, 321-351.

Willems, J. C. (1972b). Dissipative dynamical, part II: Linear systems with quadratic supply rates. Arch. Ratinal Mech. Anal, 45, 352-393.

Xu, S., Lam, J., Lin, Z., Galkowski, K., Paszke, W., Sulikowski, B., Rogers, E., \& Owens, D. H. (2003). Positive real control of two-dimensional systems: Roesser models and linear repetitive processes. International Journal of Control, 76(11), 1047-1058. 
Xu, S., Lam, J., Zou, Y., Lin, Z., \& Paszke, W. (2005). Robust $\mathcal{H}_{\infty}$ filtering for uncertain 2-D continuous systems. IEEE Transactions on Signal Processing, 53(5), 1731-1738.

Yakubovich, V. A. (1971). S-procedure in nonlinear control theory. Vestnik Leningrad University, 4, 62-77.

\section{Author Biographies}

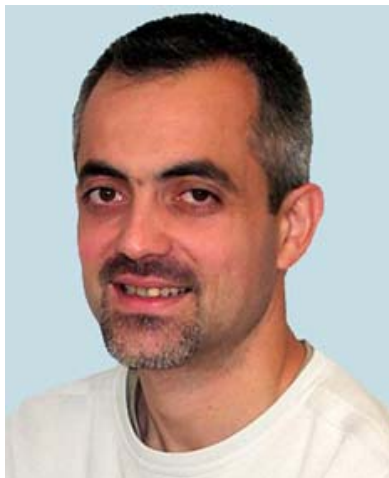

Olivier Bachelier was born in Châteauroux, France, in 1971. He received the "Diplôme d'Ingénieur" (Engineer's Degree) and the "Diplome d'Etudes Approfondies" (Masters' Degree) with specialization in control from the INSA (Institut National des Sciences Appliquées), Toulouse, France, in September 1994. From December 1995 to September 1998, he was a Ph.D. student at the LAAS (Laboratoire d'Analyse et d'Architecture des Systemes) of the CNRS (Centre National de la Recherche Scientifique) in Toulouse, France. He received the "Diplome de Doctorat" (Ph.D. degree) from INSA in September 1998. He kept on working for one year at the LAAS-CNRS. Since September 1999, he is a researcher at the LAII (Laboratoire d'Automatique et d'Informatique Industrielle) of the ESIP (Ecole Supérieure d'Ingénieurs de Poitiers). $\mathrm{He}$ is also an assistant professor at the department of Electrical Engineering of the IUT (Institut Universitaire de Technologie) of Poitiers, France. The IUT and the ESIP are parts of the University of Poitiers. His research interests are robust analysis and robust control of uncertain linear models, especially robust rootclustering.

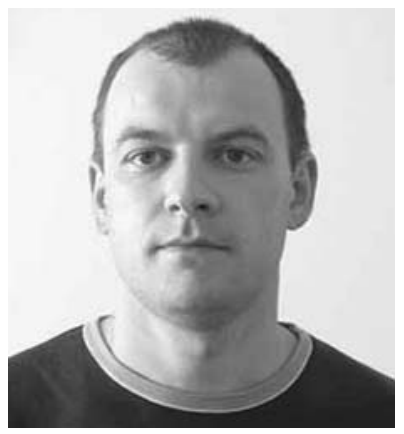

Wojciech Paszke was born in Zielona Góra, Poland, in 1975. He received his M.Sc. and Ph.D. degrees, both in electrical engineering, from the Technical University of Zielona Góra, in 2000 and 2005, respectively. After holding a position of an Assistant Professor on the Faculty of Electrical Engineering, Computer Science and Telecommunications of the University of Zielona Gora for three years, he moved to Eindhoven University of Technology, where he holds currently a postdoctoral position. His general research interests include multidimensional (n-D) systems, repetitive processes and delayed systems with particular attention to computational aspects.

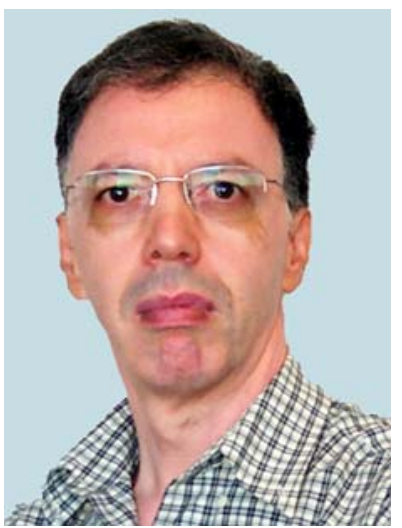

Driss Mehdi received his $\mathrm{PhD}$ in 1986 and his Habilitation in 1992 both from University of Nancy. He was appointed as Senior lecturer in 1988 at the University of Strasbourg and as Professor in 1992 at the university of Poitiers. His current interests are robust control of linear systems, descriptor systems and time delay systems. 